\title{
Enumerating some symmetry classes of rhombus tilings of holey hexagons
}

\author{
Tomack Gilmorel
}

Fakultät für Mathematik, Universität Wien, Oskar-Morgenstern-Platz 1, 1090 Vienna, Austria

\begin{abstract}
This extended abstract presents some recent (exact and asymptotic) enumerative results concerning rhombus tilings of hexagons that have had symmetrically distributed inward pointing triangles of side length 2 removed from their interiors. These results form part of a larger article that is currently available online (arXiv:1501.05772).

Résumé. Ce résumé détaillé présente quelques résultats énumératifs récents (exacts et asymptotiques) sur les pavages par losanges d'hexagones dont on a enlevé des triangles de côté 2 placés symétriquement et pointant vers l'intérieur. Ces résultats sont extraits d'un article plus développé disponible actuellement en ligne (arXiv:1501.05772).
\end{abstract}

Keywords: Non-intersecting paths, holey hexagons, rhombus tilings, enumeration.

\section{Introduction}

Consider the triangular lattice $\mathscr{T}$, that is, the lattice consisting of unit equilateral triangles drawn so that one of the sets of lattice lines is vertical. Then a unit rhombus on $\mathscr{T}$ corresponds to the pairing of two unit triangles of $\mathscr{T}$ that share exactly one edge. Let $H_{a, b, c}$ denote the hexagonal sub-region of $\mathscr{T}$, centred at some origin $O$, with side lengths $a, b, c, a, b, c$ (going counter-clockwise from southwest edge, see Figure 1 (left)). Then a rhombus tiling of $H_{a, b, c}$ corresponds to a perfect matching between all unit triangles contained within the interior of $H_{a, b, c}$. A holey hexagon, $H_{a, b, c} \backslash T$, is a hexagon $H_{a, b, c}$ that has had a set of triangles, $T$, removed from its interior. See Figure 1 (middle) for an example of a holey hexagon.

One important motivation for considering rhombus tilings of holey hexagons, as evidenced by Mihai Ciucu in his 2008 PNAS survey paper [2], is the apparent link between tilings of these regions and twodimensional electrostatics. Denote by $H_{n}=H_{n, n, n}$ the regular hexagon on $\mathscr{T}$. Suppose $T$ is a set of unit triangles contained in the interior of $H_{n}$. Then the correlation function (sometimes also referred to as the interaction) of the holes is defined to be

$$
\omega=\lim _{n \rightarrow \infty} \frac{M\left(H_{n} \backslash T\right)}{M\left(H_{n}\right)}
$$

\footnotetext{
†tomack.gilmore@univie.ac.at

Research supported by the Austrian Science Foundation (FWF), grant F50-N15, in the framework of the Special Research Program "Algorithmic and Enumerative Combinatorics".

1365-8050 @ 2015 Discrete Mathematics and Theoretical Computer Science (DMTCS), Nancy, France
} 
where $M(H)$ denotes the total number of tilings of $H$. Note that in order for this definition to make sense, any collection of holes must consist of an equal number of left and right pointing unit triangles, otherwise the number of rhombus tilings of $H_{n} \backslash T$ is 0 [i). It was conjectured by Ciucu [2] that as the distance between the set of holes in $T$ and the origin (that is, the centre of $H_{n}$ ) becomes very large, the asymptotics captured by the correlation function are governed by Coulomb's laws of electrostatics. More precisely, the asymptotics of the rhombus-mediated interaction of holes captured by this correlation function is given, up to a multiplicative constant, by the exponential of the negative of the electrostatic energy of the two-dimensional system of physical charges obtained by viewing each hole as a point charge of magnitude and sign. This magnitude and sign is specified by a statistic for each hole, namely the difference between the left and right pointing unit triangles that comprise each hole $e^{(\text {ii) }}$. More recent work concerning the interaction of a hole with a free boundary by Ciucu and Krattenthaler [5] draws further parallels between vertically symmetric tilings of holey hexagons and physical phenomena. Theorem 2.1 presented in Section 2 below is an analogue of the enumerative result found in [5]. The remainder of this section is dedicated to stating some known enumeration results for tilings of hexagons that contain no holes followed by a more explicit definition of the holey hexagons that are of particular interest.

The enumeration of rhombus tilings of hexagons (in equivalent forms and guises) has a relatively long history. The story begins with MacMahon [10] in the early twentieth century. At the time MacMahon was concerned with enumerating the number of plane partitions that fit inside an $a \times b \times c$ box, however there is a straightforward bijection between the three-dimensional representation of such plane partitions (as cubes stacked into the corner of a box) and two-dimensional hexagons covered in unit rhombi. MacMahon successfully proved the following theorem.

Theorem 1.1 (MacMahon) The total number of rhombus tilings of the hexagon $H_{a, b, c}$ is given by

$$
T(a, b, c)=\prod_{i=1}^{a} \prod_{j=1}^{b} \prod_{k=1}^{c} \frac{i+j+k-1}{i+j+k-2} .
$$

MacMahon also conjectured a formula for the number of symmetric plane partitions that fit inside an $a \times a \times b$ box. This was later proved by Andrews [1], and simultaneously (in an equivalent form, though published much later) by Gordon [8]. Under the same bijection as before, such symmetric plane partitions correspond to rhombus tilings of $H_{a, a, b}$ (from now on denoted simply by $H_{a, b}$ ) that are symmetric with respect to its vertical symmetry axis, that is, the vertical line that intersects the origin of $H_{a, b}$.

Theorem 1.2 (Andrews/Gordon) The number of vertically symmetric tilings of $H_{a, b}$ is

$$
S T(a, b)=\prod_{i=1}^{a} \frac{2 i+b-1}{2 i-1} \prod_{1 \leq i<j \leq a} \frac{i+j+b-1}{i+j-1} .
$$

One other symmetry class of plane partitions that is of particular interest is the set of transposecomplementary plane partitions. These correspond to rhombus tilings of the hexagon $H_{a, 2 b}$ that are symmetric with respect to the horizontal symmetry axis, that is, the axis that intersects the mid-points of the vertical sides of $H_{a, 2 b}$. The following formula is due to Proctor [13].

(i) Ciucu has provided further methods for determining the interaction between holes that do not fit this criteria, however for the purpose of this article the given definition will suffice.

(ii) Observe that for our definition of the correlation function to make sense, the sum of the point charges of the holes must be 0 . 

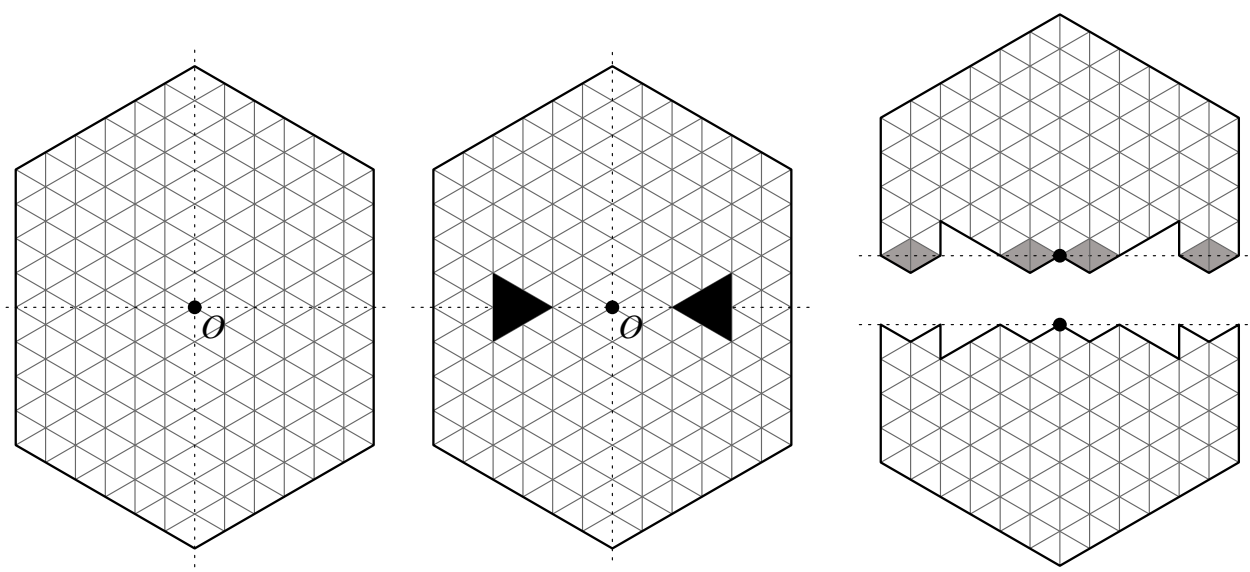

Fig. 1: Left: the hexagonal region $H_{6,6,8}$ on the triangular lattice $\mathscr{T}$. Middle: the holey hexagon $H_{6,8} \backslash\left(\triangleright_{4} \cup \triangleleft_{4}\right)$. Right: (above) the weighted region $H_{6,8}^{+} \backslash\left(\triangleright_{4} \cup \triangleleft_{4}\right)$, where the shaded rhombi have a weight of 2; (below) the lower half region $H_{6,8}^{-} \backslash\left(\triangleright_{4} \cup \triangleleft_{4}\right)$.

Theorem 1.3 (Proctor) The number of horizontally symmetric tilings of $H_{a, 2 b}$ is

$$
T C(a, 2 b)=\left(\begin{array}{c}
a+b-1 \\
a-1
\end{array}\right) \prod_{i=1}^{a-2} \prod_{j=i}^{a-2} \frac{2 b+i+j+1}{i+j+1} .
$$

The above three theorems concern hexagons that contain no holes, so the enumeration of various symmetry classes of rhombus tilings of holey hexagons may then be viewed as generalisations of these plane partition problems. An explicit definition of the holey hexagons of interest follows.

Suppose we have a hexagon $H_{n, 2 m}$ centred at some origin $O$. Let $\triangleright_{k}$ denote the right pointing triangle of side length 2 positioned symmetrically about the horizontal symmetry axis of $H_{n, 2 m}$ such that its vertical edge is at lattice distance $k$ to the left of $O$. Define the left pointing triangle $\triangleleft_{k}$ analogously. Then $H_{n, 2 m} \backslash\left(\triangleright_{k} \cup \triangleleft_{k}\right)$ denotes the hexagon $H_{n, 2 m}$ from which two inward pointing triangles of side length 2 have been removed with their vertical edges at lattice distance $k$ either side of the origin (see Figure1(middle)).

Remark 1 Note that in any such hexagon, $k$ must have the same parity as $n$.

From now on assume that $n, m$ and $k$ are positive integers, and also that $n$ and $k$ have the same parity. The results presented in this paper enumerate (exactly and asymptotically) vertically symmetric and horizontally symmetric rhombus tilings of $H_{n, 2 m} \backslash\left(\triangleright_{k} \cup \triangleleft_{k}\right)$ (see Figure 2 and Figure 3 as well as enumerating tilings of the complete region $H_{n, 2 m} \backslash\left(\triangleright_{k} \cup \triangleleft_{k}\right)$.

The next section states the main results of this abstract. Section 3 discusses the representation of rhombus tilings of holey hexagons as families of non-intersecting lattice paths, leading to the expression of numbers of sets of tilings as determinants of particular matrices. In Section 4 these determinants are evaluated by explicitly stating the unique $L U$-decomposition of the matrices from Section 3 . In the final 
section some asymptotic formulas for the interaction of these holes are briefly stated and discussed and compared with those of [5] .

Note that while the results presented here pertain to holey hexagons that have vertical edges of even length, analogous results for hexagons with vertical edges of odd length have also been discovered and may be found in [7] alongside full proofs of the theorems presented below.

\section{Main Results}

Let $l$ denote the free boundary that coincides with the vertical symmetry axis of the hexagon $H_{n, 2 m} \backslash$ $\left(\triangleright_{k} \cup \triangleleft_{k}\right)$. The line $l$ is "free" in the sense that unit rhombi are permitted to protrude across it. Let $V_{n, 2 m} \backslash \triangleright_{k}$ denote the region to the left of $l$ (see Figure 2). Then enumerating vertically symmetric tilings of $H_{n, 2 m} \backslash\left(\triangleright_{k} \cup \triangleleft_{k}\right)$ corresponds to enumerating rhombus tilings of $V_{n, 2 m} \backslash \triangleright_{k}$ (see Figure 2 (left and middle-left)).

Theorem 2.1 The number of rhombus tilings of $V_{n, 2 m} \backslash \triangleright_{k}$, denoted $M\left(V_{n, 2 m} \backslash \triangleright_{k}\right)$, is

where

$$
\left[\sum_{s=1}^{m} B_{n, k}(s) \cdot D_{n, k}(s)\right] \times S T(n, 2 m),
$$

$$
\begin{aligned}
B_{n, k}(s)= & \frac{(-1)^{s+1}(-k+n+1) !(n+s-1) !(n+2 s-1) !\left(\frac{k}{2}+\frac{n}{2}+s-2\right) !}{(s-1) !\left(\frac{n}{2}-\frac{k}{2}\right) !\left(\frac{k}{2}+\frac{n}{2}-1\right) !(2 n+2 s-1) !\left(-\frac{k}{2}+\frac{n}{2}+s\right) !}, \\
D_{n, k}(s)= & \frac{(-1)^{s+1}(2 s-2) !(n-k) !(n+s-1) !\left(\frac{k}{2}+\frac{n}{2}+s-2\right) !}{(s-1) !\left(\frac{n}{2}-\frac{k}{2}\right) !\left(\frac{k}{2}+\frac{n}{2}-1\right) !(n+2 s-2) !\left(-\frac{k}{2}+\frac{n}{2}+s\right) !} \\
& +\frac{2(-1)^{s+1}(2 s-2) !(n-k) !(n+s) !\left(\frac{k}{2}+\frac{n}{2}+s-2\right) !}{(s-2) !\left(\frac{n}{2}-\frac{k}{2}\right) !\left(\frac{k}{2}+\frac{n}{2}\right) !(n+2 s-2) !\left(-\frac{k}{2}+\frac{n}{2}+s\right) !}
\end{aligned}
$$

Remark 2 The above result is analogous to that of Ciucu and Krattenthaler [5] in the sense the hole in $V_{n, 2 m}$ has been "flipped" and points towards the centre.

Now let $H_{n, 2 m}^{-} \backslash\left(\triangleright_{k} \cup \triangleleft_{k}\right)$ denote the lower half of $H_{n, 2 m} \backslash\left(\triangleright_{k} \cup \triangleleft_{k}\right)$, that is, the region lying beneath the zig-zag line that proceeds just below the horizontal symmetry axis of $H_{n, 2 m} \backslash\left(\triangleright_{k} \cup \triangleleft_{k}\right)$ (see Figure 1 (right-lower)). Then the number of rhombus tilings of $H_{n, 2 m}^{-} \backslash\left(\triangleright_{k} \cup \triangleleft_{k}\right)$ corresponds to the number of horizontally symmetric tilings of $H_{n, 2 m} \backslash\left(\triangleright_{k} \cup \triangleleft_{k}\right)$ (see Figure 3).

Theorem 2.2 The number of rhombus tilings of $H_{n, 2 m}^{-} \backslash\left(\triangleright_{k} \cup \triangleleft_{k}\right)$, is

$$
\left[\sum_{s=1}^{m} B_{n, k}^{\prime}(s) \cdot D_{n, k}^{\prime}(s)\right] \cdot T C(n, 2 m),
$$

where

$$
\begin{aligned}
B_{n, k}^{\prime}(t) & =\frac{(-1)^{t-1}(t+n-2) !(2 t+n-1) !(n-k) !\left(t+\frac{k}{2}+\frac{n}{2}-2\right) !}{2(t-1) !(2 t+2 n-3) !\left(\frac{n}{2}-\frac{k}{2}\right) !\left(\frac{k}{2}+\frac{n}{2}-1\right) !\left(t-\frac{k}{2}+\frac{n}{2}\right) !} \\
D_{n, k}^{\prime}(s) & =\frac{(-1)^{s+1}(2 s) !(s+n-1) !(n-k) !\left(s+\frac{k}{2}+\frac{n}{2}-2\right) !}{2(s !)(2 s+n-2) !\left(\frac{k}{2}+\frac{n}{2}-1\right) ! \frac{n-k}{2} !\left(s-\frac{k}{2}+\frac{n}{2}\right) !}
\end{aligned}
$$


Denote by $H_{n, 2 m}^{+} \backslash\left(\triangleright_{k} \cup \triangleleft_{k}\right)$ the upper half of $H_{n, 2 m} \backslash\left(\triangleright_{k} \cup \triangleleft_{k}\right)$, that is, the region lying above $H_{n, 2 m}^{-} \backslash\left(\triangleright_{k} \cup \triangleleft_{k}\right)$, and suppose that the unit rhombi lying along the bottom edge of $H_{n, 2 m}^{+} \backslash\left(\triangleright_{k} \cup \triangleleft_{k}\right)$ have weight 2 (see Figure 1 (right-upper)). By Ciucu's Matchings Factorization Theorem [3] it follows that

$$
M\left(H_{n, 2 m} \backslash\left(\triangleright_{k} \cup \triangleleft_{k}\right)\right)=M\left(H_{n, 2 m}^{-} \backslash\left(\triangleright_{k} \cup \triangleleft_{k}\right)\right) \cdot M_{w}\left(H_{n, 2 m}^{+} \backslash\left(\triangleright_{k} \cup \triangleleft_{k}\right)\right),
$$

where $M_{w}\left(H_{n, 2 m}^{+} \backslash\left(\triangleright_{k} \cup \triangleleft_{k}\right)\right)$ denotes the number of weighted tilings of the region $H_{n, 2 m}^{+} \backslash\left(\triangleright_{k} \cup \triangleleft_{k}\right)$. An argument as to why (2.1) holds may be found in [4]. Hence enumerating the total number of tilings of the region $H_{n, 2 m} \backslash\left(\triangleright_{k} \cup \triangleleft_{k}\right)$ reduces to enumerating tilings of the region $H_{n, 2 m}^{+} \backslash\left(\triangleright_{k} \cup \triangleleft_{k}\right)$ which may then be combined via 2.1] with the previous result for horizontally symmetric tilings.

Theorem 2.3 The number of weighted rhombus tilings of $H_{n, 2 m}^{+} \backslash\left(\triangleright_{k} \cup \triangleleft_{k}\right)$ is

$$
\left[\sum_{s=1}^{m} B_{n, k}(s) \cdot E_{n, k}(s)\right] \cdot S T(n, 2 m),
$$

where $B_{n, k}(s)$ is defined as in Theorem 2.1 and

$$
E_{n, k}(s)=\frac{(-1)^{s+1}(2 s-2) !(-k+n+1) !(n+s-1) !\left(\frac{k}{2}+\frac{n}{2}+s-2\right) !}{(s-1) !\left(\frac{n}{2}-\frac{k}{2}\right) !\left(\frac{k}{2}+\frac{n}{2}-1\right) !(n+2 s-2) !\left(-\frac{k}{2}+\frac{n}{2}+s\right) !} .
$$

The next corollary follows immediately.

Corollary 2.4 The total number of rhombus tilings of the region $H_{n, 2 m} \backslash\left(\triangleright_{k} \cup \triangleleft_{k}\right)$ is

$$
\left[\sum_{s=1}^{m} B_{n, k}^{\prime}(s) \cdot D_{n, k}^{\prime}(s)\right] \times\left[\sum_{t=1}^{m} B_{n, k}(t) \cdot E_{n, k}(t)\right] \times T(n, 2 m, n) .
$$

The following section describes a standard translation of rhombus tilings of hexagons into families of non-intersecting paths on the square lattice, which may be counted by taking determinants of certain matrices.

\section{From Rhombus Tilings to Non-intersecting Lattice Paths}

Any rhombus tiling of $H_{n, 2 m} \backslash\left(\triangleright_{k} \cup \triangleleft_{k}\right)$ (and consequently any tiling of the half-hexagons defined in the previous section) may be represented as unique sets of lattice paths across dimers. Since these paths across dimers consist of steps in one of two directions they may in turn be translated into families of nonintersecting lattice paths consisting of north and east steps on the square lattice, $\mathbb{Z}^{2}$. Then enumerating rhombus tilings of $H_{n, 2 m} \backslash\left(\triangleright_{k} \cup \triangleleft_{k}\right)$ reduces to enumerating families of non-intersecting lattice paths, for which there already exist many well-known methods.

Under this bijection, tilings of the region $V_{n, 2 m} \backslash \triangleright_{k}$ correspond precisely to families of non-intersecting paths that begin at the set of start points $A=\bigcup_{i=1}^{2 m} A_{i}$, where $A_{i}=(-i, i)$, and end at a set of points $I=\left\{(x, y) \in \mathbb{Z}^{2}: x+y=n\right\} \cup\left\{t_{1}, t_{2}\right\}$, with the requirement that $t_{1}=((n-k) / 2-m,(n-k) / 2+m)$ and $t_{2}=((n-k) / 2-m-1,(n-k) / 2+m+1)$ must be included as end points (see Figure 2). According to Stembridge [14, Theorem 3.2] (and also Okada [11]), the number of families of such paths may be expressed in terms of a Pfaffian. 

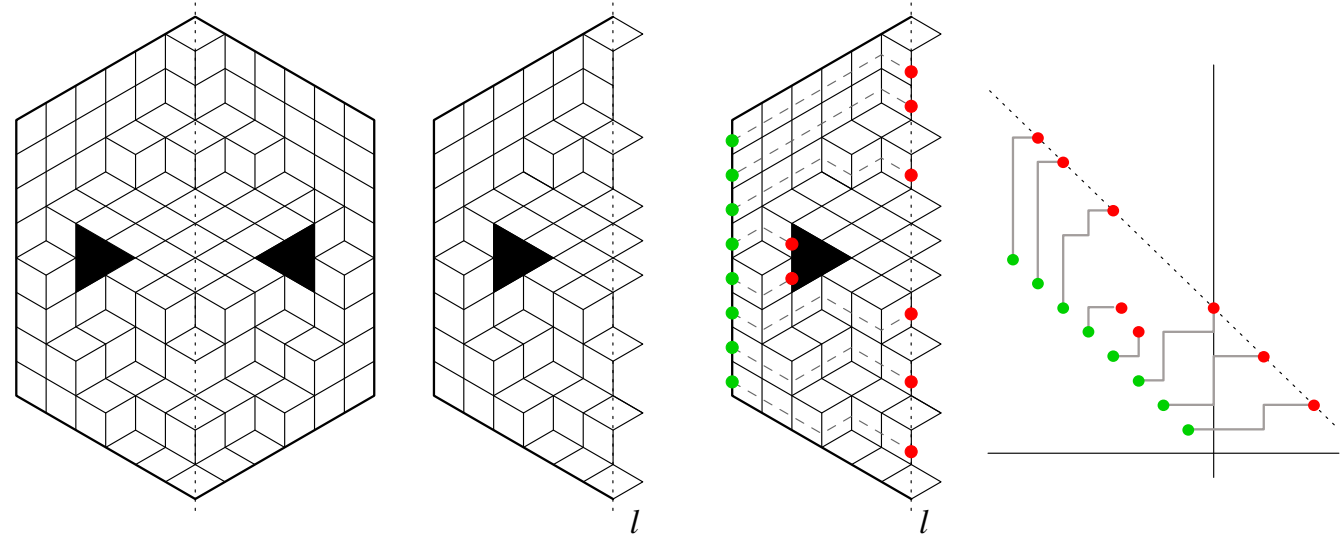

Fig. 2: From left to right: a vertically symmetric tiling of $H_{6,8} \backslash\left(\triangleright_{4} \cup \triangleleft_{4}\right)$; the corresponding tiling of the region $V_{6,8} \backslash \triangleright_{4}$; the set of lattice paths across dimers corresponding to this tiling of $V_{6,8} \backslash \triangleright_{4}$; and the family of nonintersecting lattice paths representing this tiling of $V_{6,8} \backslash \triangleright_{4}$ in $\mathbb{Z}^{2}$.

Proposition 3.1 The number of vertically symmetric tilings of the hexagon $H_{n, 2 m} \backslash\left(\triangleright_{k} \cup \triangleleft_{k}\right)$ is the Pfaffian of the $(2 m+2) \times(2 m+2)$ skew-symmetric matrix $F$ with $(i, j)$-entries given by

$$
F_{i, j}= \begin{cases}\sum_{r=i-j+1}^{j-i}\left(\begin{array}{c}
2 n \\
n+r
\end{array}\right), & 1 \leq i<j \leq 2 m \\
\left(\begin{array}{l}
n-k \\
(n-k) / 2-m+i
\end{array}\right), & i \in\{1, \ldots, 2 m\}, j=2 m+1, \\
\left(\begin{array}{l}
n-k \\
(n-k) / 2-m-1+i
\end{array}\right), & i \in\{1, \ldots, 2 m\}, j=2 m+2, \\
0, & 2 m+1 \leq i<j \leq 2 m+2 .\end{cases}
$$

In a similar way it is possible to express the number of tilings of $H_{n, 2 m}^{-} \backslash\left(\triangleright_{k} \cup \triangleleft_{k}\right)$ and $H_{n, 2 m}^{+} \backslash\left(\triangleright_{k} \cup \triangleleft_{k}\right)$ as families of non-intersecting paths which may in turn be expressed as a determinant. These cases are more straightforward since tilings of such regions correspond to families of non-intersecting paths between a set of start points and a set of fixed end points.

Consider first the region $H_{n, 2 m}^{-} \backslash\left(\triangleright_{k} \cup \triangleleft_{k}\right)$. By applying the classic theorem of Lindström, Gessel and Viennot [6] it is possible to deduce the following.

Proposition 3.2 The number of horizontally symmetric tilings of $H_{n, 2 m} \backslash\left(\triangleright_{k} \cup \triangleleft_{k}\right)$ is $(-\operatorname{det}(G))$, where $G$ is the $(m+1) \times(m+1)$ matrix with $(i, j)$-entries given by

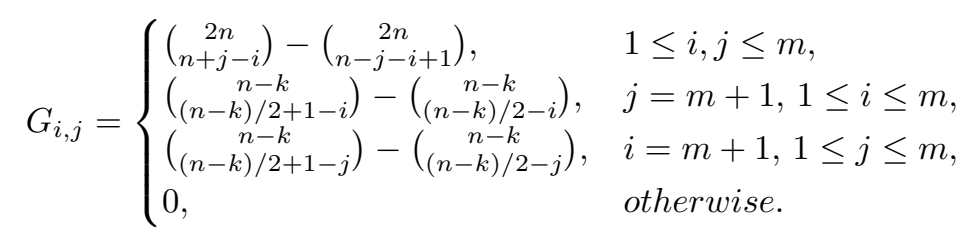



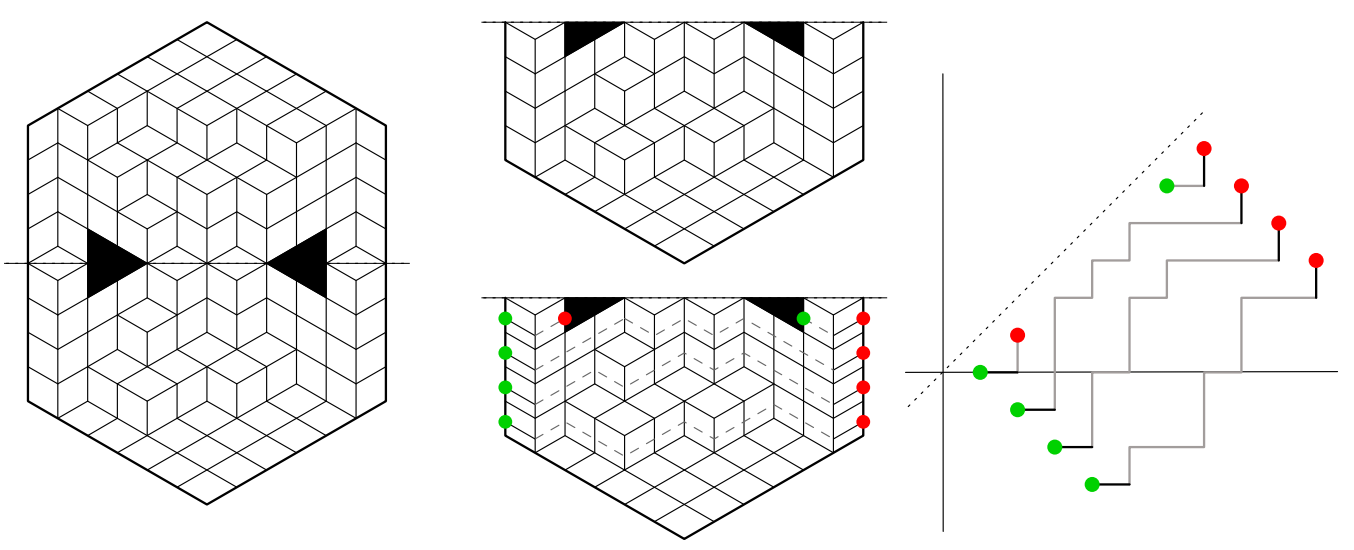

Fig. 3: Left: a horizontally symmetric tiling of $H_{6,8} \backslash\left(\triangleright_{4} \cup \triangleleft_{4}\right)$. Middle (upper): the corresponding tiling of $H_{6,8}^{-} \backslash\left(\triangleright_{4} \cup \triangleleft_{4}\right)$. Middle (lower): the corresponding set of lattice paths across dimers, where forced dimers are denoted by the thick black lines on the vertical sides. Right: the corresponding family of lattice paths in $\mathbb{Z}^{2}$, here forced dimers correspond to forced east and north steps in black.

The number of tilings of $H_{n, 2 m}^{+} \backslash\left(\triangleright_{k} \cup \triangleleft_{k}\right)$ may be calculated in almost exactly the same way, with the exception that any path $P$ that intersects the line $y=x$ at $t(P)$-many points is treated with a weight of $2^{t(P)}$ (see [4]). Again by applying Lindström, Gessel and Viennot's theorem it is possible to derive the following proposition:

Proposition 3.3 The number of weighted tilings of the region $H_{n, 2 m}^{+} \backslash\left(\triangleright_{k} \cup \triangleleft_{k}\right)$ is given by $\left(-\operatorname{det}\left(G^{+}\right)\right)$, where $G^{+}$is the $(m+1) \times(m+1)$ matrix with $(i, j)$-entries given by

$$
G_{i, j}^{+}= \begin{cases}\left(\begin{array}{c}
2 n \\
n-i-j+1
\end{array}\right)+\left(\begin{array}{c}
2 n \\
n-k+1 \\
(n-k) / 2+i
\end{array}\right), & 1 \leq i, j \leq m, \\
\left(\begin{array}{c}
n-k+1 \\
(n-k) / 2+j
\end{array}\right), & j=m+1,1 \leq i \leq m, \\
0, & i=m+1,1 \leq j \leq m,\end{cases}
$$

Theorem 2.2 and Theorem 2.3 are derived by finding the unique $L U$-decomposition of the corresponding matrices defined above. In the case of vertically symmetric tilings it is convenient to first reduce the matrix $F$ to a much smaller matrix $F^{*}$ by way of an extension of Gordon's Lemma [9]. Then Theorem 2.1 is obtained by finding the unique $L U$-decomposition of the matrix $F^{*}$.

\section{Evaluation of Determinants}

Consider the $(2 m+2) \times(2 m+2)$ matrix $F$ from Proposition 3.1 in the previous section. This matrix satisfies the conditions of the following lemma, which is an extension of that of Gordon [9].

Lemma 4.1 For a positive integer $m$ and a non-negative integer $l$, let $A$ be a $(2 m+2 l) \times(2 m+2 l)$ skew-symmetric matrix of the form 


$$
A=\left(\begin{array}{cc}
X & Y \\
-Y^{t} & Z
\end{array}\right)
$$

for which the following properties hold:

1. $X$ is a $2 m \times 2 m$ matrix such that $X=\left(x_{j-i}\right)_{1 \leq i, j \leq 2 m}$ and $x_{j, i}=-x_{i, j}$;

2. $Z$ is a $2 l \times 2 l$ matrix satisfying $z_{i, j}+z_{i+l, j}+z_{i, j+l}=0$ for $1 \leq i, j \leq l$, and $z_{j, i}=-z_{i, j}$;

3. $Y$ is a $2 m \times 2 l$ matrix such that:

$$
y_{i, j}= \begin{cases}y_{2 m-i, j}, & 1 \leq i \leq m, 1 \leq j \leq l, \\ y_{2 m+1-i, j-l}, & 1 \leq i \leq 2 m, l+1 \leq j \leq 2 l .\end{cases}
$$

Then

$$
\operatorname{Pf}(A)=(-1)\left(\left(\begin{array}{c}
m+l \\
2
\end{array}\right)+\left(\begin{array}{c}
m \\
2
\end{array}\right)\right) \operatorname{det}(B),
$$

where $B$ is an $(m+l) \times(m+l)$ matrix of the form

$$
\left(\begin{array}{cc}
\widehat{X} & \widehat{Y}_{1} \\
\widehat{Y}_{2} & \widehat{Z}
\end{array}\right)
$$

the block matrices of which are defined by

$$
\begin{array}{rr}
(\widehat{X})_{i, j}=x_{i+j-1}+x_{i+j-3}+\cdots+x_{|i-j|+1} & \text { for } 1 \leq i, j \leq m, \\
\left(\widehat{Y}_{1}\right)_{i, j}=\sum_{r=0}^{i-1}\left(y_{m+1-i+2 r, j}-y_{m+i-2 r, j}\right) & \text { for } 1 \leq i \leq m \text { and } 1 \leq j \leq l, \\
\left(\widehat{Y}_{2}\right)_{i, j}=\sum_{r=0}^{j-1}\left(y_{j+m-2 r, i}+y_{m+1-j+2 r, i}\right) & \text { for } 1 \leq i \leq l \text { and } 1 \leq j \leq m, \\
(\widehat{Z})_{i, j}=z_{i, j+l}+z_{i+l, j+l} & \text { for } 1 \leq i, j \leq l .
\end{array}
$$

Applying this lemma directly to the matrix $F$ defined in Proposition 3.1 results in the following expression for the signed Pfaffian of $F$,

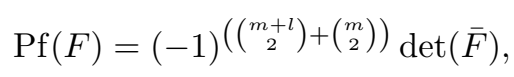

where $\bar{F}$ is the $(m+1) \times(m+1)$ matrix with $(i, j)$-entries given by

$$
\bar{F}_{i, j}=\left\{\begin{array}{ll}
F_{i+j-1}+F_{i+j-3}+\cdots+F_{|i-j|+1}, & 1 \leq i, j \leq m \\
\sum_{r=0}^{i-1}\left(F_{m+1-i+2 r, 2 m+1}-F_{m+i-2 r, 2 m+1}\right), & 1 \leq i \leq m, j=m+1, \\
\sum_{r=0}^{j-1}\left(F_{j+m-2 r, 2 m+1}+F_{m+1-j+2 r, 2 m+1}\right), & i=m+1,1 \leq j \leq m, \\
0, & \text { otherwise. }
\end{array} .\right.
$$

Subtracting row $(m-i)$ from row $(m+1-i)$ for $i \in\{1, \ldots, m-1\}$ (and performing the same operations on the columns) of $\bar{F}$ and then reversing the order of rows 1 to $m$ to construct a new matrix $F^{*}$, one sees that

$$
M\left(V_{n, 2 m} \backslash \triangleright_{k}\right)=\left|\operatorname{det}\left(F^{*}\right)\right|,
$$


where $F^{*}$ is the $(m+1) \times(m+1)$ matrix with $(i, j)$-entry given by

$$
F_{i, j}^{*}= \begin{cases}\left(\begin{array}{c}
2 n \\
n-i-j+1
\end{array}\right)+\left(\begin{array}{c}
2 n \\
n+i-j
\end{array}\right), & 1 \leq i, j \leq m, \\
\left(\frac{4(i-1)}{k-n}+\frac{2 i-1}{i+(n-k) / 2}\right)\left(\begin{array}{c}
n-k \\
(n-k) / 2-i+1
\end{array}\right), & j=m+1,1 \leq i \leq m, \\
\left(\begin{array}{c}
n-k+1 \\
(n-k) / 2+j
\end{array}\right), & i=m+1,1 \leq j \leq m, \\
0, & \text { otherwise }\end{cases}
$$

Note that the positive part of $\operatorname{det}\left(F^{*}\right)$ is taken in 4.1 since we are counting families of paths.

Theorem 4.2 The $(m+1) \times(m+1)$ matrix $F^{*}$ described above has LU-decomposition

$$
F^{*}=L^{*} \cdot U^{*}
$$

where $L^{*}$ has the form

$$
L_{i, j}^{*}= \begin{cases}A_{n}(i, j), & 1 \leq j \leq i \leq m \\ B_{n, k}(j), & i=m+1,1 \leq j \leq m \\ 1, & i=j=m+1 \\ 0, & \text { otherwise }\end{cases}
$$

and $U^{*}$ has the form

$$
U_{i, j}^{*}= \begin{cases}C_{n}(i, j), & 1 \leq i \leq j \leq m \\ D_{n, k}(i), & j=m+1,1 \leq i \leq m \\ -\sum_{s=1}^{m} B_{n, k}(s) \cdot D_{n, k}(s), & i=j=m+1 \\ 0, & \text { otherwise }\end{cases}
$$

where

$$
\begin{aligned}
A_{n}(i, j) & =\frac{(n) !(i+j-2) !(2 j+n-1) !}{(2 j-2) !(i-j) !(-i+j+n) !(i+j+n-1) !}, \\
B_{n, k}(j)= & \frac{(-1)^{j+1}(j+n-1) !(2 j+n-1) !(n-k+1) !(j+(k+n) / 2-2) !}{(j-1) !(2 j+2 n-1) !((n-k) / 2) !((k+n) / 2-1) !(j+(n-k) / 2) !} \\
C_{n}(i, j)= & \frac{(n) !(i+j-2) !(2 i+2 n-1) !}{(j-i) !(2 i+n-2) !(i-j+n) !(i+j+n-1) !}, \\
D_{n, k}(i)= & \frac{(-1)^{i+1}(2 i-2) !(i+n-1) !(n-k) !(i+(k+n) / 2-2) !}{(i-1) !(2 i+n-2) !((n-k) / 2) !((k+n) / 2-1) !(i+(n-k) / 2) !} \\
& \quad+\frac{2(-1)^{i+1}(2 i-2) !(i+n) !(n-k) !(i+(k+n) / 2-2) !}{(i-2) !(2 i+n-2) !((n-k) / 2) !((k+n) / 2) !(i+(n-k) / 2) !}
\end{aligned}
$$

The proof of the above theorem reduces to showing that a number of hypergeometric identities hold. These can be verified by using your favourite implementation of the Gosper-Zeilberger algorithm (see for example [12]). 
It is easy to see that $A_{n, k}(i, i)=1$ for $i \in\{1, \ldots, m\}$, so the $L U$-decomposition of $F^{*}$ above is unique. Theorem 2.1 then follows from taking the positive product of the diagonal entries of $U^{*}$.

Since $F_{i, j}^{*}=G_{i, j}^{+}$for $i \in\{1, \ldots, m+1\}, j \in\{1, \ldots, m\}$ and $i=j=m+1$, a little further work yields the following:

Lemma 4.3 The $(m+1) \times(m+1)$ matrix $G^{+}$has LU-decomposition

$$
G^{+}=L^{*} \cdot U^{+}
$$

where $L^{*}$ is as before and

$$
U_{i, j}^{+}= \begin{cases}U_{i, j}^{*}, & 1 \leq i \leq j \leq m \\ E_{n, k}(i), & 1 \leq i \leq m, j=m+1, \\ -\sum_{s=1}^{m} B_{n, k}(s) \cdot E_{n, k}(s), & i=j=m+1\end{cases}
$$

where $E_{n, k}(s)$ is defined to be

$$
E_{n, k}(s)=\frac{(-1)^{s+1}(2 s-2) !(n-k+1) !(n+s-1) !((k+n) / 2+s-2) !}{(s-1) !((n-k) / 2) !((k+n) / 2-1) !(n+2 s-2) !((n-k) / 2+s) !} .
$$

Again, since $L^{*}$ contains $1 \mathrm{~s}$ along its diagonal, Theorem 2.3 follows immediately by taking the positive product of the diagonal entries of $U^{+}$.

Remark 3 Observe that in Proposition 3.3 the holes in $H_{n, 2 m}^{+} \backslash\left(\triangleright_{k} \cup \triangleleft_{k}\right)$ correspond to the last row and column of $G^{+}$. Hence the determinant of the $m \times m$ sub-matrix $G^{\prime}$, where $G_{i, j}^{\prime}=G_{i, j}^{+}$for $1 \leq i, j \leq m$, counts the number of tilings of $H_{n, 2 m}^{+}$, the same weighted region that contains no holes. It is not hard to convince oneself that $G^{\prime}=L^{\prime} \cdot U^{\prime}$ where $L^{\prime}=L_{i, j}^{*}$ and $U^{\prime}=U_{i, j}^{*}$ for $1 \leq i, j \leq m$. Hence it follows that $M_{w}\left(H_{n, 2 m}^{+}\right)=S T(n, 2 m)=M\left(V_{n, 2 m}\right)$ and so by 2.1), $M\left(H_{n, 2 m}\right)=M\left(V_{n, 2 m}\right) \cdot M\left(H_{n, 2 m}^{-}\right)$, which is a special case of the results found in [4]. No bijective proof of this appealing factorisation exists, however the relation observed between $S T(n, 2 m)$ and $T C(n, 2 m)$, namely,

$$
\left(\begin{array}{c}
n+m-1 \\
m-1
\end{array}\right) \cdot M\left(V_{n, 2 m}\right)=\left(\begin{array}{c}
2 n+2 m-1 \\
2 m-1
\end{array}\right) \cdot M\left(H_{n, 2 m}^{-}\right)
$$

might shed new light on the inner workings of such a bijection.

In order to derive an expression for the total number of tilings of $H_{n, 2 m} \backslash\left(\triangleright_{k} \cup \triangleleft_{k}\right)$ it suffices to determine the $L U$-decomposition of the matrix $G$. Then by applying 2.1) we may construct a formula for $M\left(H_{n, 2 m} \backslash\left(\triangleright_{k} \cup \triangleleft_{k}\right)\right)$.

Theorem 4.4 The matrix $G$ whose determinant counts the number of horizontally symmetric tilings of $H_{n, 2 m} \backslash\left(\triangleright_{k} \cup \triangleleft_{k}\right)$ has LU-decomposition

$$
G=L \cdot U
$$

where

$$
L_{i, j}= \begin{cases}A_{n}^{\prime}(i, j), & 1 \leq j \leq i \leq m, \\ B_{n, k}^{\prime}(j), & i=m+1,1 \leq j \leq m, \\ 1, & i=j=m+1 \\ 0, & \text { otherwise. }\end{cases}
$$


and $U$ is given by

$$
U_{i, j}= \begin{cases}C_{n}^{\prime}(i, j), & 1 \leq i \leq j \leq m, \\ D_{n, k}^{\prime}(i), & j=m+1,1 \leq i \leq m, \\ -\sum_{s=1}^{m} B_{n, k}^{\prime}(s) \cdot D_{n, k}^{\prime}(s), & i=j=m+1 \\ 0, & \text { otherwise }\end{cases}
$$

where

$$
\begin{aligned}
A_{n}^{\prime}(s, t) & =\frac{n !(s+t-2) !(2 t+n-1) !(2 s-1)}{(2 t-1) !(s-t) !(t-s+n) !(s+t+n-1) !}, \\
B_{n, k}^{\prime}(t) & =\frac{(-1)^{t-1}(t+n-2) !(2 t+n-1) !(n-k) !\left(t+\frac{k}{2}+\frac{n}{2}-2\right) !}{2(t-1) !(2 t+2 n-3) !\left(\frac{n}{2}-\frac{k}{2}\right) !\left(\frac{k}{2}+\frac{n}{2}-1\right) !\left(t-\frac{k}{2}+\frac{n}{2}\right) !} \\
C_{n}^{\prime}(s, t) & =\frac{(2 t-1) n !(s+t-2) !(2 s+2 n-2) !}{(t-s) !(2 s+n-2) !(s-t+n) !(s+t+n-1) !} \\
D_{n, k}^{\prime}(s) & =\frac{(-1)^{s+1}(2 s) !(s+n-1) !(n-k) !\left(s+\frac{k}{2}+\frac{n}{2}-2\right) !}{2(s !)(2 s+n-2) !\left(\frac{k}{2}+\frac{n}{2}-1\right) ! \frac{n-k}{2} !\left(s-\frac{k}{2}+\frac{n}{2}\right) !}
\end{aligned}
$$

Once again a straightforward calculation shows that $A_{n}^{\prime}(i, i)=1$ for $i \in\{1, \ldots, m\}$ and hence Theorem 2.2 follows immediately from taking the positive product of the diagonal entries of $U$. Combining the above formula with Theorem 2.2 by way of 2.1) gives Corollary 2.4.

\section{Asymptotics}

Denote by $\omega_{H}(k)$ the correlation function

$$
\omega_{H}(k)=\lim _{n \rightarrow \infty} \frac{M\left(H_{n, n} \backslash\left(\triangleright_{k} \cup \triangleleft_{k}\right)\right)}{M\left(H_{n, n}\right)} .
$$

Let $\omega_{V}(k)$ and $\omega_{H^{-}}(k)$ denote the analogous correlation functions obtained by replacing $H_{n, 2 m} \backslash\left(\triangleright_{k} \cup \triangleleft_{k}\right)$ in (5.1) with $V_{n, n} \backslash \triangleright_{k}$ and $H_{n, n}^{-} \backslash\left(\triangleright_{k} \cup \triangleleft_{k}\right)$ respectively.

Theorem 5.1 As $k \rightarrow \infty$ the correlation functions $\omega_{H}, \omega_{V}$ and $\omega_{H^{-}}$are asymptotically

1. $\omega_{H}(k) \sim\left(\frac{\sqrt{3}}{2 \pi \mathrm{d}\left(\triangleright_{k}, \triangleleft_{k}\right)}\right)^{2}$,

2. $\omega_{V}(k) \sim \frac{3}{4 \pi \mathrm{d}\left(\triangleright_{k}, l\right)}$,

3. $\omega_{H^{-}}(k) \sim \frac{3}{2 \pi \mathrm{d}\left(\triangleright_{k}, \triangleleft_{k}\right)}$,

where $\mathrm{d}(a, b)$ denotes the Euclidean distance between $a$ and $b$.

Remark 4 The first correlation result above is in keeping with the conjecture of Ciucu [2]. The second, concerning the interaction of a right pointing hole with the free boundary $l$, is particularly surprising. The correlation function for the same region but with the hole "flipped" and pointing away from $l$ was determined asymptotically by Ciucu and Krattenthaler [5] to be $1 /\left(4 \pi \mathrm{d}\left(\triangleleft_{k}, l\right)\right)$. As can be seen above, flipping the hole so that it points in the opposite direction amounts to multiplying the (asymptotic) correlation function by a factor of 3 . Any reason as to why such a discrepancy should occur remains a complete mystery. 


\section{Acknowledgements}

The author would like to thank Christian Krattenthaler and the referees for their careful reading of this submission and helpful comments.

\section{References}

[1] G. E. Andrews. Plane partitions I: The MacMahon conjecture, Studies in foundations and combinatorics, G.-C. Rota ed., Adv. in Math. Suppl. Studies, Vol. 1 (1978), pp. 131-150.

[2] M. Ciucu. Dimer packings with gaps and electrostatics, Proc. Natl. Acad. Sci. USA, Vol. 105 (2008), pp. 2766-2772.

[3] M. Ciucu. Enumeration of perfect matchings in graphs with reflective symmetry, J. Combin. Theory Ser. A, Vol. 77 (1997), pp. 67-97.

[4] M. Ciucu \& C. Krattenthaler. A factorization theorem for lozenge tilings of a hexagon with triangular holes, arxiv preprint, arXiv:1403.3323 (2014), available at http://arxiv.org/ abs/1403.3323

[5] M. Ciucu \& C. Krattenthaler. The interaction of a gap in a two-dimensional dimer system, Commun. Math. Physics. 302 (2011), pp. 253-289.

[6] I. M. Gessel \& X. G. Viennot. Determinants, paths, and plane partitions, preprint (1989), available at http://people.brandeis.edu/ gessel/homepage/papers/pp.pdf

[7] T. Gilmore. Three interactions of holes in two dimensional dimer systems, arxiv preprint, arXiv:1501.05772, available at http://arxiv.org/abs/1501.05772

[8] B. Gordon. A Proof of the Bender-Knuth Conjecture, Pacific J. Math., Vol. 108 (1) (1983), pp. 99-113.

[9] B. Gordon. Notes on plane partitions V, J. Comb. Theory Ser. B, Vol. 11 (2) (1971), pp. 157-168.

[10] P. A. MacMahon. Combinatory Analysis, Vol. 2, Cambridge University Press, 1916; (reprinted Chelsea, New York, 1960).

[11] S. Okada. On the generating functions for certain classes of plane partitions, J. Comb. Theory Ser. A, Vol. 1 (1989), pp. 1-23.

[12] P. Paule \& M. Schorn. A Mathematica version of Zeilberger's algorithm for proving binomial coefficient identities, J. Symbolic Comput., Vol. 20 (1995), pp. 673-698.

[13] R. A. Proctor. Bruhat lattices, plane partitions generating functions, and minuscule representations, Europ. J. Combin., Vol. 5 (1984), pp. 331-350.

[14] J. R. Stembridge. Nonintersecting paths, pfaffians and plane partitions, Advances in Math., Vol. 83 (1990), pp. 96-131. 